\title{
The Temperature Stabilization System of CUORICINO: An Array of Macro Bolometers
}

\author{
C. Arnaboldi, C. Bucci, S. Capelli, P. Gorla, E. Guardincerri, A. Nucciotti, G. Pessina, S. Pirro, and M. Sisti
}

\begin{abstract}
We present our circuit solution for the implementation of a very simple temperature stabilization system of an array of 62 large mass bolometric detectors, CUORICINO. The implemented instrument exploits one of the front-end channels to close the feedback loop between a thermistor and a heather, both of them located on the detector holder in the refrigerating system. A very compact layout was developed that avoids any possible presence of EMI interference, which would otherwise be introduced if a commercial instrument were adopted. The stabilized temperature is obtained exploiting the offset correcting circuit of the remotely programmable front-end channel to autogenerate the reference operating point for the feedback loop. Thanks to the adopted configuration, the instability of the cryogenic apparatus, which induced a drift corresponding to about $50 \mathrm{keV} / \mathrm{day}$, was reduced to less than about $0.5 \mathrm{keV} / \mathrm{day}$. To model the system behavior, we have reformulated the bolometer theory of operation using the concept of a feedback network. The obtained results will be shown.
\end{abstract}

Index Terms-Bolometers, closed-loop systems, cryogenic electronics, cryogenics, proportional control, stability criteria.

\section{INTRODUCTION}

$\mathbf{E}$ XPERIMENTS in high energy physics that must run for a long time always need a stabilization system that assures the constancy of the energy conversion gain. The simplest way to maintain a constant gain with high accuracy is to locate a known source of particles close to the detector, allowing a continuous calibration. Unfortunately, often it is not possible to easily tag every signal induced by the calibrating source. This effect may degrade the accuracy in the study of the process under investigation by adding spurious events to the final energy spectrum.

An experiment performed with an array of bolometers is not an exception to this problem. CUORICINO [1] is an array composed of $62 \mathrm{TeO}_{2}$ bolometric crystals, featuring $42 \mathrm{~kg}$ of total mass. Its aim is to study the Double beta decay, $\mathrm{D} \beta \beta$, of ${ }^{130} \mathrm{Te}$. Every detector of the array is composed of a $\mathrm{TeO}_{2}$ crystal with volume either $5 \times 5 \times 5 \mathrm{~cm}^{3}(790 \mathrm{~g})$ or $3 \times 3 \times 6 \mathrm{~cm}^{3}(340 \mathrm{~g})$, depending on the location within the array. On every crystal a Ge

Manuscript received November 26, 2004; revised May 3, 2005.

C. Arnaboldi, S. Capelli, A. Nucciotti, G. Pessina, S. Pirro, and M. Sisti are with Sezione di Milano dell'INFN and Dipartimento di Fisica, Università di Milano-Bicocca, I-20126 Milano, Italy (e-mail: Gianluigi.Pessina@mib.infn.it).

C. Bucci is with Laboratori Nazionali del Gran Sasso, I-67010 Assergi (L'Aquila), Italy.

P. Gorla is with the Laboratorio de Fisica Nuclear y Altas Energias, Universidàd de Zaragoza, 50009 Zaragoza, Spain.

E. Guardincerri is with Dipartimento di Fisica and Sezione di Genova dell'INFN, Università di Genova, I-16146 Genova, Italy.

Digital Object Identifier 10.1109/TNS.2005.856758
NTD [2] thermistor is glued down, that converts the temperature increase following the absorption of an impinging particle into an electrical signal. An adequate signal to noise ratio requires very small operating temperatures, close to $10 \mathrm{mK}$.

The $\mathrm{D} \beta \beta$ is a very rare process. Its study requires very long measurement times and a very low level of radioactive background. Therefore, a very accurate stabilization system that does not add background is a stringent requirement. The procedure presently adopted to stabilize CUORICINO is as follows. On a periodic basis, usually once per month, a source of energetic particles is put close to the detector to perform an absolute calibration of the energy scale. Between these periodic calibrations, the monitoring of the baseline of every bolometer permits accounting for the temperature of the detector itself and its sensitivity. This condition is met if the front-end does not add drift, as is the case for CUORICINO [3]. In addition to the baseline monitoring, we inject a voltage which develops a power pulse across a special heater [4] glued on every crystal in a proper location, far from the thermistor. The heating pulse simulates the signal generated by an incoming particle. A very stable multi-output pulse generator is used to perform this task [5]. The periodic illumination of the detector array with a particle source together with the baseline monitoring and the pulse calibration has given CUORICINO a very good temperature stabilization [6].

To further improve the stability, we have verified that the detector array needs a very high precision temperature stabilization. For this reason a Proportional, Integrative, Derivative (PID) instrument should be used to stabilize the detector holder temperature, inside the refrigerator system. A standard, commercial, solution might be adopted. Some drawbacks of such an arrangement are to be considered. We need to stabilize the temperature around $10 \mathrm{mK}$ using a thermometer with the same level of sensitivity as the Ge NTD thermistors we use, having hundreds of $\mathrm{M} \Omega$ resistance value. The standard procedure to stabilize temperature consists on balancing an AC resistance bridge cascaded with a PID controller that closes the loop on a heater. These devices are, in general, range limited to few $\mathrm{M} \Omega$, due to the presence of stray shunting capacitances. In addition, the usual frequency range of this AC bias (tens of $\mathrm{Hz}$ ) is larger than the bandwidth of our detector signals.

Another approach is based on the lock-in technique [7], [8], that, as for the $\mathrm{AC}$ resistance bridge, needs an alternate signal to be applied to the thermistor. As a consequence, the lock-in technique is very efficient with bolometers having small value resistances, too. 


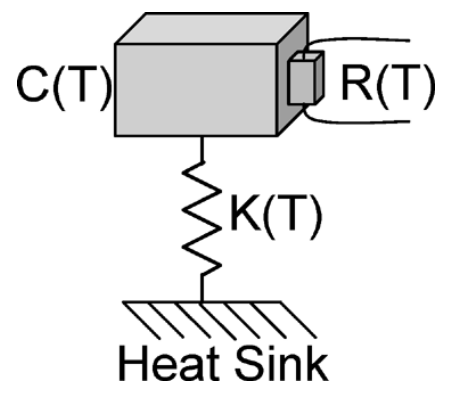

Fig. 1. Simplified thermal model of a bolometer.

To overcome the above limitations to large value thermistors, we have designed and built a very simple circuit able to stabilize the temperature by simply exploiting one channel of the front-end itself to close the loop between the thermometer and the heating resistors. The very important result obtained is that EMI interferences are not injected. In addition the thermometer is operated in the same way as any detector of the array, biased at DC. The operating point is chosen by simply trimming the offset of the channel that, in this circumstance, becomes the reference voltage of the error correcting loop. The set point is easily obtained thanks to the remote programmability of any channel of the set [9].

In the next sections a description of the theory of operation of the stabilization system of the cryogenic array, the complete circuit solution and the experimental results will be shown in detail.

\section{THEORY OF OPERATION OF THE STABILIZATION SYSTEM}

\section{A. Principle of Operation of a Bolometer}

A bolometer is composed of an absorbing crystal to which a thermistor resistor is attached. At very low temperatures the heat capacity of the absorbing crystal becomes vanishing, according to the Debye law. The thermistor is constructed in order to have high sensitivity. We use Ge NTD thermistors in our experiments, that are well modeled by the function [10], [11]:

$$
R(T)=R_{o} \exp \left(\sqrt{\frac{T_{\mathrm{o}}}{\mathrm{T}}}\right) .
$$

Our devices have $R_{o}$ of the order of a few $\Omega$ and $T_{o}$ of about $3 \mathrm{~K}$. Using these two values in (1), the thermistor operating points are found around $100 \mathrm{M} \Omega$ for temperatures between $5 \mathrm{mK}$ and 10 $\mathrm{mK}$. A very simplified thermal model of a bolometric detector is shown in Fig. 1.

In Fig. 2 a simulation, performed with Simulink ${ }^{1}$ of the characteristics of a bolometer read by a Ge NTD thermistor is shown for different base temperatures, from $5 \mathrm{mK}$ to $10 \mathrm{mK}$. The shape of the I-V curves depends on the value of the thermal conductance toward the heat sink. In Fig. 2 the thermal conductance was supposed mainly due to the gold wires bonded on the thermistor and to the bonds themselves. Their resulting temperature dependence follows the law:

$$
\mathrm{K}(\mathrm{T})=\frac{\Delta \mathrm{P}}{\Delta \mathrm{T}}=\mathrm{K}_{\mathrm{o}} \mathrm{T}^{\beta}
$$

${ }^{1}$ Simulink is a trademark of The MathWorks, Inc.

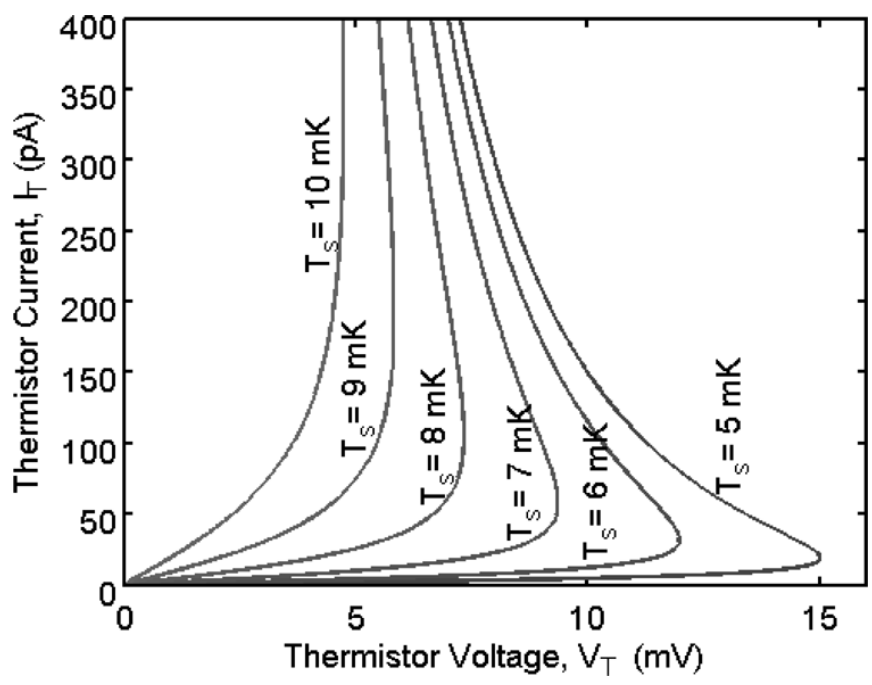

Fig. 2. I-V characteristics at different temperatures of a Ge NTD thermistor having $\mathrm{T}_{\mathrm{o}}=3.3 \mathrm{~K}, \mathrm{R}_{\mathrm{o}}=1.15 \Omega$, a thermal conductance toward the heat sink of $40 \mathrm{~T}^{2.4} \mu \mathrm{K} / \mathrm{W}$, and a background power of $400 \mathrm{pW}$.

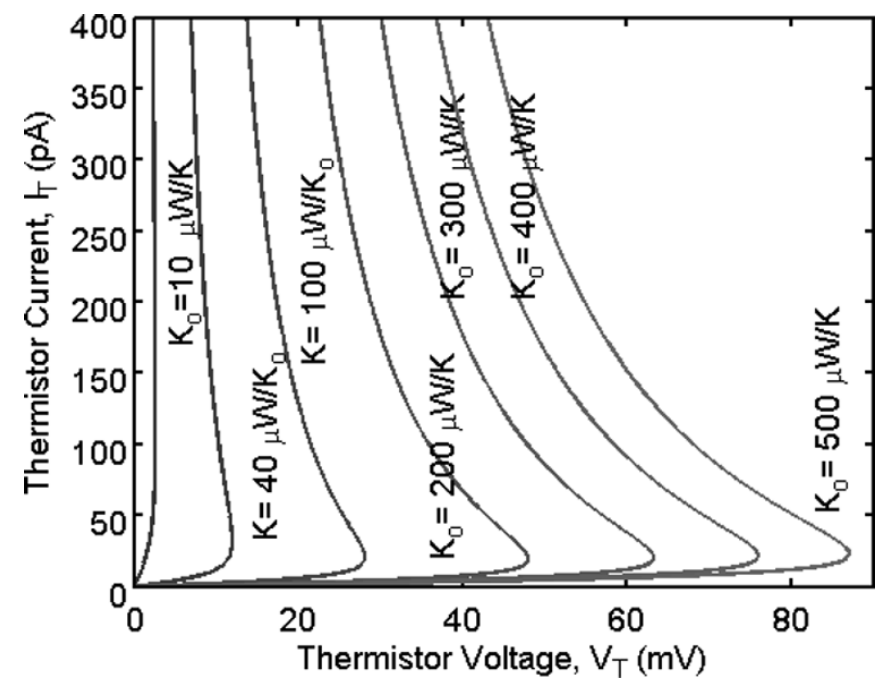

Fig. 3. I-V characteristics for different values of the parameter $K_{o}$ of (2) for a Ge NTD thermistor having To $=3.3 \mathrm{~K}, \mathrm{Ro}_{\mathrm{O}}=1.15 \Omega$, a background power of $400 \mathrm{pW}$, and at a heat sink temperature of $6 \mathrm{mK}$.

where $\beta$ equals 2.4 [12] and $\mathrm{P}(\mathrm{T})$ is the dissipated power. The background power can rarely be neglected in these applications. In the simulation shown it was assumed equal to $400 \mathrm{pW}$.

The larger the thermal conductance, the more the I-V characteristics are expected to be linear over a larger voltage range. This is visible in Fig. 3 where the simulations have $K_{0}$ of (2) as the parameter of the I-V curves. Both the behaviors in Fig. 2 and Fig. 3 are a consequence of electrothermal feedback [13]-[16]. When a bias is applied, a power flows in the thermal conductance, which raises the bolometer temperature. The temperature increase changes the thermistor characteristics that, in turn, modify the dissipated power itself, closing the feedback loop.

On every I-V curve of Fig. 2 or Fig. 3 the current and the voltage are temperature dependent and correlated to the thermal conductance $\mathrm{K}(\mathrm{T})$. The electrothermal feedback imposes a balance between the temperature and the dissipated power. At any point on any $\mathrm{I}-\mathrm{V}$ curve of Fig. 2 the thermistor voltage, $\mathrm{V}_{\mathrm{T}}(\mathrm{T})$, the thermistor current $\mathrm{I}_{\mathrm{T}}(\mathrm{T})$, the dissipated power $\mathrm{P}_{\mathrm{T}}(\mathrm{T})$, and 
the temperature T must be related. Exploiting (2), we can determine the relation between the thermistor operating point and its dynamic characteristics:

$$
\mathrm{K}(\mathrm{T})=\frac{\Delta\left(\mathrm{V}_{\mathrm{T}}(\mathrm{T}) \mathrm{I}_{\mathrm{T}}(\mathrm{T})\right)}{\Delta \mathrm{T}} .
$$

For a semiconductor thermistor it can be written:

$\mathrm{K}(\mathrm{T})=\frac{\Delta \mathrm{P}_{\mathrm{T}}}{\Delta \mathrm{T}}=\frac{\Delta \mathrm{P}_{\mathrm{T}}}{\Delta \mathrm{R}} \frac{\Delta \mathrm{R}}{\Delta \mathrm{T}}=-\alpha \frac{\Delta \mathrm{P}_{\mathrm{T}}}{\Delta \mathrm{R}}, \quad \alpha=-\frac{\Delta \mathrm{R}}{\Delta \mathrm{T}}, \quad \alpha>0$.

Using (4) and the relation $\Delta \mathrm{R}=\Delta(\mathrm{V} / \mathrm{I})$ in (3) it results that:

$$
\mathrm{K}(\mathrm{T})=-\alpha \mathrm{I}_{\mathrm{T}}^{2} \frac{\mathrm{Z}+\mathrm{R}}{\mathrm{Z}-\mathrm{R}}, \quad \mathrm{Z}=\frac{\Delta \mathrm{V}_{\mathrm{T}}}{\Delta \mathrm{I}_{\mathrm{T}}}, \quad \mathrm{R}(\mathrm{T})=\frac{\mathrm{V}_{\mathrm{T}}}{\mathrm{I}_{\mathrm{T}}} .
$$

$\mathrm{Z}$ being the differential impedance on the I-V curve. Since from the first law of thermodynamic $\mathrm{K}(\mathrm{T})$ must be positive, it follows that, under any conditions, $\mathrm{R} \geq \mathrm{Z}$ must be always satisfied. In addition, if the thermal conductance is large, or there is a good thermal contact, then $\mathrm{Z}$ tends to be close to $\mathrm{R}$, and the I- $\mathrm{V}$ curves become straight lines.

Equation (5) sets a relation between the thermistor current and its static, small signal (differential) characteristics.

A scheme for the small signal model of the electrothermal effect is shown in Fig. 4 [16]-[18]. We first consider the feedback effect suppressed by connecting the (not actually present) virtual switch $\mathrm{SW}$ to the heat sink. We would expect that, as a response to a small input power $\Delta \mathrm{P}_{\mathrm{i}}$, an open loop change of the temperature $\Delta \mathrm{T}, \Delta \mathrm{T}_{\mathrm{OL}}$, results in:

$$
\Delta \mathrm{T}_{\mathrm{OL}}=\frac{\Delta \mathrm{P}_{\mathrm{i}}}{\mathrm{K}+\mathrm{j} \omega \mathrm{C}},
$$

and, consequently, a change is also induced for the thermistor resistance $\mathrm{R}(\mathrm{T}), \Delta \mathrm{R}=-\alpha \Delta \mathrm{T}_{\mathrm{OL}}$. The electrical power undergoes a change proportional to $\Delta \mathrm{R}$ that, in turn, is proportional to $\Delta \mathrm{T}_{\mathrm{OL}}: \Delta \mathrm{P}_{\mathrm{F}}=-\mathrm{A} \alpha \Delta \mathrm{T}_{\mathrm{OL}}$. When $\mathrm{SW}$ is connected to the heat sink this quantity is proportional to the loop gain, $\operatorname{LG}(\omega)$, therefore:

$$
\mathrm{LG}(\omega)=\frac{\Delta \mathrm{P}_{\mathrm{F}}}{\Delta \mathrm{P}_{\mathrm{i}}}=-\frac{\alpha \mathrm{A}}{\mathrm{K}+\mathrm{j} \omega \mathrm{C}} .
$$

By considering the virtual switch SW connected to the node at the temperature $\Delta \mathrm{T}$, the overall transfer function becomes:

$$
\Delta \mathrm{T}=\frac{\text { Open LoopGain }}{1-\text { Loop Gain }} \Delta \mathrm{P}_{\mathrm{i}}=\frac{1}{\mathrm{~K}+\mathrm{j} \omega \mathrm{C}} \frac{1}{1-\mathrm{LG}(\omega)} \Delta \mathrm{P}_{\mathrm{i}} .
$$

As it is known for a feedback system having a dominant pole, the open loop time constant, $\mathrm{C}(\mathrm{T}) / \mathrm{K}(\mathrm{T})$ for the present case, is reduced by a factor proportional to the value of $1-\mathrm{LG}(0)=$ $1+\alpha \mathrm{A} / \mathrm{K}$.

The term A in (7) can be extracted from the electrical mesh of Fig. 4 (it has to be remarked that, when $V_{\text {BIAS }}$ does not change, the operating point jumps from one curve to another in response to $\Delta \mathrm{P}_{\mathrm{i}}$ in Fig. 2):

$$
\mathrm{A}=\frac{\Delta \mathrm{P}_{\mathrm{EL}}}{\Delta \mathrm{R}}=\frac{\mathrm{Z}_{\mathrm{L}}-\mathrm{R}}{\mathrm{Z}_{\mathrm{L}}+\mathrm{R}} \mathrm{I}^{2}, \quad \mathrm{I}=\frac{\mathrm{V}_{\mathrm{BIAS}}}{\mathrm{Z}_{\mathrm{L}}+\mathrm{R}} .
$$

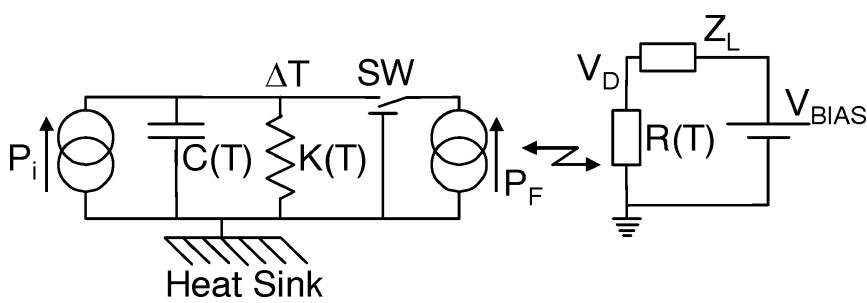

Fig. 4. Electrothermal model of a bolometer.

It is interesting to observe that the feedback can be positive and the detector may break into oscillation at any frequency for which $\left|Z_{L}\right|$ is less than $R$. Or, the system transfer function may have two or more poles if $Z_{\mathrm{L}}$ is complex, i.e., as a typical case $\mathrm{Z}_{\mathrm{L}}=\mathrm{R}_{\mathrm{L}} \| \mathrm{C}_{\mathrm{L}}$, where $\mathrm{C}_{\mathrm{L}}$ may be a parasitic shunting capacitance.

Merging together (5) through (9), we obtain:

$$
\begin{aligned}
\Delta \mathrm{T} & =\frac{\Delta \mathrm{P}_{\mathrm{i}}}{\mathrm{C}} \frac{1}{\mathrm{j} \omega+\frac{\mathrm{K}}{\mathrm{C}}\left[1+\frac{\mathrm{Z}_{\mathrm{L}}-\mathrm{R}}{\mathrm{Z}_{\mathrm{L}}+\mathrm{R}} \frac{\alpha \mathrm{I}^{2}}{\mathrm{~K}}\right]} \\
& =\frac{\Delta \mathrm{P}_{\mathrm{i}}}{\mathrm{C}} \frac{1}{\mathrm{j} \omega+\frac{\mathrm{K}}{\mathrm{C}}\left[\frac{2 \mathrm{R}}{\mathrm{Z}+\mathrm{R}} \frac{\mathrm{Z}_{\mathrm{L}}+\mathrm{Z}}{\mathrm{Z}_{\mathrm{L}}+\mathrm{R}}\right]} .
\end{aligned}
$$

The feedback has the effect to speed up the recovery of the detector to its stationary condition. The larger the bias current, the larger the applied feedback and the smaller the resulted recovery time.

If we calculate $\Delta V_{D}$ from the output mesh of Fig. 4 , remembering $\mathrm{V}_{\text {BIAS }}$ constant, we obtain the voltage developed across the thermistor:

$$
\begin{aligned}
\Delta \mathrm{V}_{\mathrm{D}} & =\frac{\mathrm{Z}_{\mathrm{L}}}{\mathrm{Z}_{\mathrm{L}}+\mathrm{R}} \mathrm{I} \Delta \mathrm{R} \\
& =-\frac{\mathrm{Z}_{\mathrm{L}}}{\mathrm{Z}_{\mathrm{L}}+\mathrm{R}} \frac{\alpha \mathrm{I}}{\mathrm{C}} \frac{1}{\mathrm{j} \omega+\frac{\mathrm{K}}{\mathrm{C}}\left[\frac{2 \mathrm{R}}{\mathrm{Z}+\mathrm{R}} \frac{\mathrm{Z}_{\mathrm{L}}+\mathrm{Z}}{\mathrm{Z}_{\mathrm{L}}+\mathrm{R}}\right]} \Delta \mathrm{P}_{\mathrm{i}} .
\end{aligned}
$$

We can also evaluate the expected dependence of $\mathrm{Z}$ on frequency, $Z(\omega)$. If we apply a high frequency excitation to the bolometer, the heat capacity will respond with a thermal short circuit to the heat sink. Hence $Z(\infty)=R(T)$ and, since $\mathrm{Z}(0)=\mathrm{Z} \leq \mathrm{R}(\mathrm{T}), \mathrm{Z}(\omega)$ is expected to increase with frequency, showing an inductive behavior. The mathematical interpretation of this can be made considering a small variation of the bias voltage $V_{\text {BIAS }}, \Delta V_{\text {BIAS }}$, in Fig. 4 . From the output mesh we found:

$$
\Delta \mathrm{V}_{\mathrm{D}}=\frac{\mathrm{Z}_{\mathrm{L}}}{\mathrm{Z}_{\mathrm{L}}+\mathrm{R}} \mathrm{I} \Delta \mathrm{R}+\frac{\mathrm{R}}{\mathrm{Z}_{\mathrm{L}}+\mathrm{R}} \Delta \mathrm{V}_{\mathrm{BIAS}} .
$$

The electrical power $\Delta \mathrm{P}_{\mathrm{EL}}$ is now given by the sum of two terms:

$$
\Delta \mathrm{P}_{\mathrm{EL}}=-\frac{\mathrm{Z}_{\mathrm{L}}-\mathrm{R}}{\mathrm{Z}_{\mathrm{L}}+\mathrm{R}} \mathrm{I}^{2} \alpha \Delta \mathrm{T}+\frac{2 \mathrm{R}}{\mathrm{Z}_{\mathrm{L}}+\mathrm{R}} \mathrm{I} \Delta \mathrm{V}_{\mathrm{BIAS}}
$$

where the first term is $\Delta \mathrm{P}_{\mathrm{F}}$, see (9), while the second is the input power of (8). 
Considering the dependence of $\Delta \mathrm{R}$ on $\Delta \mathrm{T}$ and that $\Delta \mathrm{I}=$ $\left(\Delta \mathrm{V}_{\text {BIAS }}-\Delta \mathrm{V}_{\mathrm{D}}\right) / \mathrm{R}_{\mathrm{L}}$, from (5), (8), (12), and (13), after a few calculations we obtain:

$$
\mathrm{Z}(\omega)=\frac{\Delta \mathrm{V}_{\mathrm{D}}(\omega)}{\Delta \mathrm{I}(\omega)}=\mathrm{Z} \frac{1+\mathrm{j} \omega \frac{\mathrm{C}}{\mathrm{K}} \frac{\mathrm{R}+\mathrm{Z}}{2 \mathrm{Z}}}{1+\mathrm{j} \omega \frac{\mathrm{C}}{\mathrm{K}} \frac{\mathrm{R}+\mathrm{Z}}{2 \mathrm{R}}} .
$$

\section{B. Principle of Operation of the Stabilization}

So far we have described the characteristics of a bolometer. Now we apply the analyzed theory to the stabilization of a complex system such as the frame of an array of large mass bolometers, CUORICINO. The 62 detector channels of CUORICINO are mounted inside an oxygen free copper frame having a mass of about $12 \mathrm{Kg}$. At very low temperatures the heat capacity of copper, a metal, is proportional to the temperature, while that of the $\mathrm{TeO}_{2}$, a dielectric and diamagnetic material, is proportional to the third power of temperature. As a consequence the overall heat capacity of the 62 detectors is negligible in comparison to that of the metallic frame. The frame is thermally connected to the heat sink with 2 copper strips $40 \mathrm{~mm}$ long, $12 \mathrm{~mm}$ wide, and $50 \mu \mathrm{m}$ thick [19]. Both the heat capacity and the thermal conductance of the frame are relatively large.

A thermistor similar to those used for the readout of the $\mathrm{TeO}_{2}$ crystals and a $\mathrm{Si}$ resistor heater of good stability [4] are well attached to the frame at opposite locations. To stabilize the frame temperature we use the circuit diagram shown in Fig. 5. The readout of the thermistor is done by exploiting the same front-end chain used for any detector of the array [20], [21]. It consists of a pair of large value load resistors, $\mathrm{R}_{\mathrm{L}} / 2(27 \mathrm{G} \Omega$ ), a multistage programmable amplifier (PGA) and the offset adjustment circuit, all of them located at room temperature. To this standard system we add a transconductance amplifier, $\mathrm{g}_{\mathrm{m}}$, to close the feedback loop on the heater resistor, $R_{h}$.

The system of Fig. 5 is again a feedback loop. Neglecting the effect on the frame temperature of the very small power dissipated in the thermistor, (3) assumes now the form:

$$
\begin{aligned}
\mathrm{K}_{\mathrm{F}}(\mathrm{T}) & =2 \alpha\left[\mathrm{V}_{\mathrm{T}}-\mathrm{V}_{\mathrm{OFF}}\right] \mathrm{A}_{\mathrm{PGA}}^{2} \mathrm{~g}_{\mathrm{m}}^{2} \mathrm{R}_{\mathrm{h}} \frac{\mathrm{Z}_{\mathrm{T}}}{\mathrm{R}_{\mathrm{T}}-\mathrm{Z}_{\mathrm{T}}} \mathrm{I}_{\mathrm{T}} \\
& =\left[\mathrm{V}_{\mathrm{T}}-\mathrm{V}_{\mathrm{OFF}}\right] \frac{\mathrm{Z}_{\mathrm{T}}}{\mathrm{R}_{\mathrm{T}}-\mathrm{Z}_{\mathrm{T}}} \gamma \\
\text { with } \mathrm{I}_{\mathrm{T}} & =\frac{\mathrm{V}_{\mathrm{BIAS}}}{\mathrm{R}_{\mathrm{T}}+\mathrm{R}_{\mathrm{L}}} .
\end{aligned}
$$

$A_{P G A}$ is the voltage gain of the PGA amplifier, and $Z_{T}$ and $R_{T}$ are the thermistor dynamic and static impedances, respectively. The other parameters of (15) are indicated in Fig. 5. From (1) the sensitivity $\alpha$ is:

$$
\alpha=-\frac{\Delta \mathrm{R}}{\Delta \mathrm{T}}=\mathrm{R}(\mathrm{T}) \frac{1}{2 \mathrm{~T}_{\mathrm{o}}}\left(\frac{\mathrm{T}_{\mathrm{o}}}{\mathrm{T}}\right)^{\frac{3}{2}} .
$$

In our experiment the operating temperature is about $9.7 \mathrm{mK}$, starting from an heat sink temperature of about $7 \mathrm{mK}$, the bias current $\mathrm{I}_{\mathrm{T}}$ is $20 \mathrm{pA}$, and $\mathrm{R}_{\mathrm{T}}(\mathrm{T})$ is about $100 \mathrm{M} \Omega$. $\mathrm{A}_{\mathrm{PGA}}$ is $7500 \mathrm{~V} / \mathrm{V}, \mathrm{g}_{\mathrm{m}} 4 \mu \mathrm{A} / \mathrm{V}$, and $\mathrm{R}_{\mathrm{h}} 50 \mathrm{~K} \Omega$. The sensitivity $\alpha$ reaches about $10^{11} \Omega / \mathrm{K}\left(\mathrm{T}_{\mathrm{O}}=3.3 \mathrm{~K}\right)$, while the thermal conductance is $14 \mu \mathrm{W} / \mathrm{K}\left(\mathrm{K}_{\mathrm{F}}(\mathrm{T})=1.56 \times 10^{-3} \mathrm{~T} \mathrm{~W} / \mathrm{K}\right.$ for the copper strips used in CUORICINO [19]). The quantity $\mathrm{K}_{\mathrm{F}} / \gamma$ in (15)

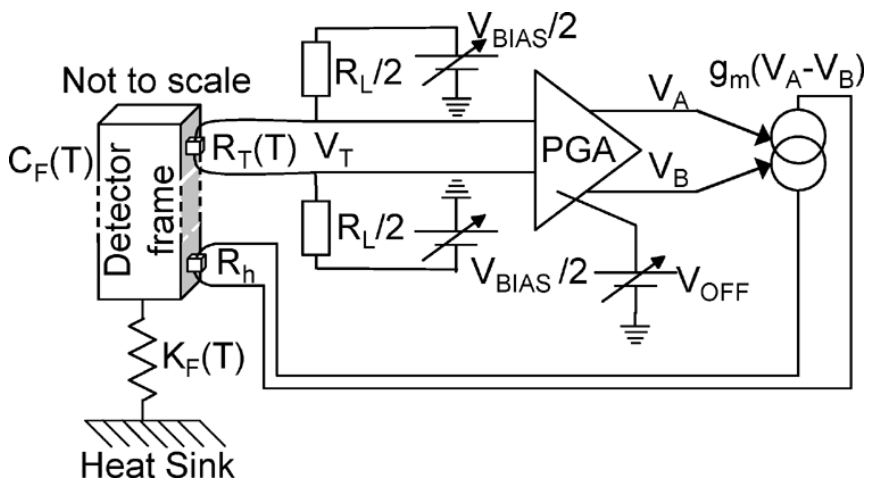

Fig. 5. Simplified schematic of the temperature stabilization system. The load resistors $R_{L}$, the amplifier PGA, and the current generators $V_{\text {OFF }}$ and $g_{m}$ are all located at room temperature.

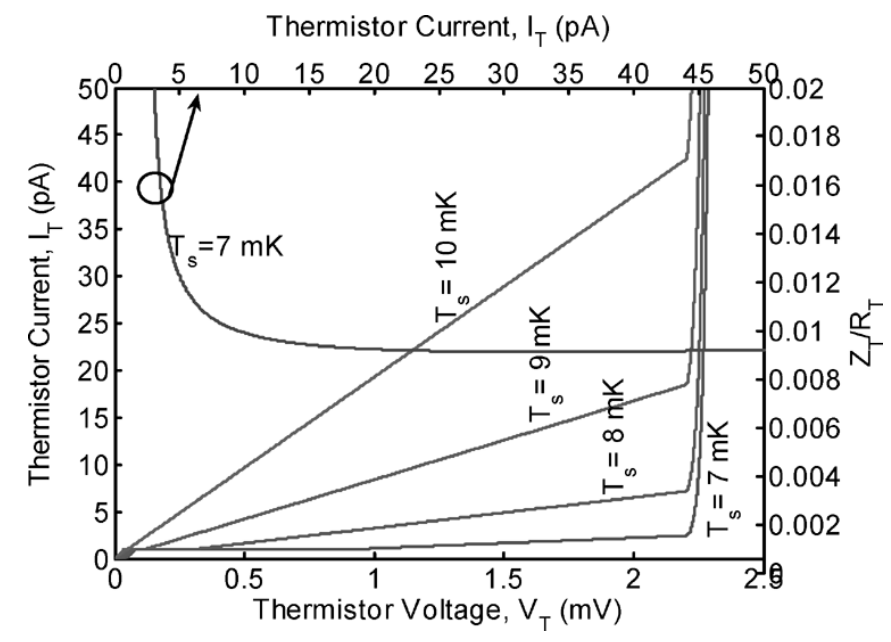

Fig. 6. I-V curves of the system of Fig. 5 at different heat sink temperatures, left and bottom axis. The ratio $Z_{T} / R_{T}$ for the temperature of $7 \mathrm{mK}$ is represented on the right and upper axis.

is about $80 \mathrm{nV}$. This means that the bias $\mathrm{V}_{\mathrm{T}}$ of the thermistor is forced to be very close to $\mathrm{V}_{\mathrm{OFF}}$, the chosen set point, apart the coefficient $\mathrm{Z}_{\mathrm{T}} / \mathrm{R}_{\mathrm{T}}$. Both $\mathrm{V}_{\mathrm{T}}$ and $\mathrm{Z}_{\mathrm{T}} / \mathrm{R}_{\mathrm{T}}$ are not independent. The reason is evident from the graphs of Fig. 2 and Fig. 3 where $Z_{T}$ is seen to become negligible when $V_{T}$ approaches constant value, as it happens in (15). In the simulations of Fig. 6 it is shown how the ratio $Z_{T} / R_{T}$ assumes small values when the feedback effect takes place. The ratio is slightly larger than 0.0092 at the operating point. It is possible to see that the I-V curves show a linear behavior until the set point $\mathrm{V}_{\mathrm{OFF}}$, of about $2.2 \mathrm{mV}$, is reached. This happens because if the current given to the thermistor develops a voltage smaller than $\mathrm{V}_{\mathrm{OFF}}$, the feedback would try, unrealistically, to lower the temperature of the heat sink. The consequence is that the loop opens, the frame temperature does not change with respect to the thermistor bias, and the thermistor has the constant value it should have at that heat sink temperature.

The above described operation is allowed by the block $\mathrm{g}_{\mathrm{m}}$ of Fig. 5, whose circuit diagram is shown in Fig. 7. Two current generators $\mathrm{A}_{\mathrm{G} 1}$ and $\mathrm{A}_{\mathrm{G} 2}$ convert the differential input voltage, amplified a factor 2 , in a current given to the heater $R_{h}$. Resistor $R_{2}$ and $R_{3}$ are equal. As a consequence the value of $g_{m}=$ $I_{h} /\left(V_{A}-V_{B}\right)$ equals $2 / R_{1}$, where $R_{1}$ has been chosen to be 


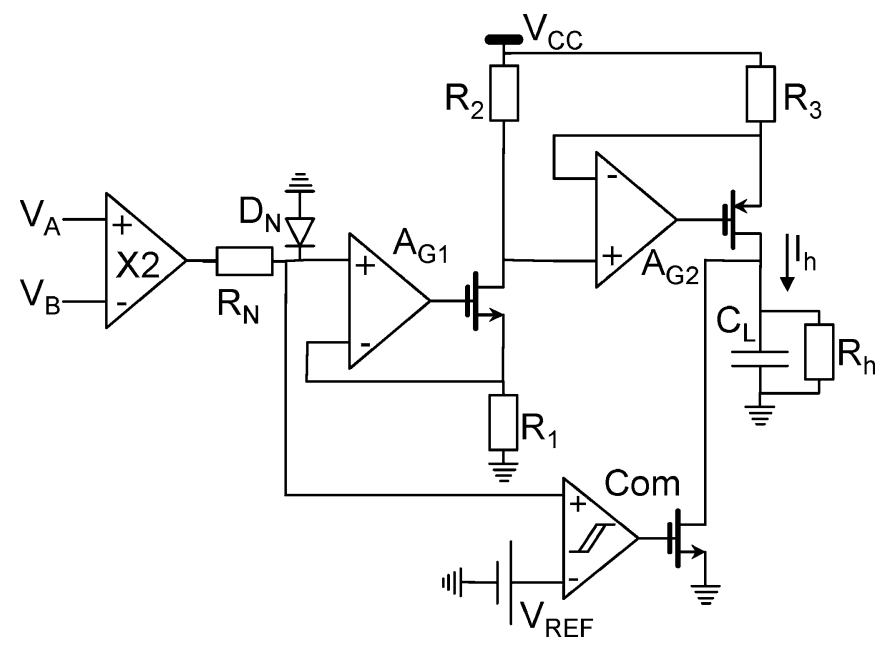

Fig. 7. Expansion of the block element $g_{m}$ of Fig. 5.

$10 \mathrm{R}_{\mathrm{h}}$. The circuit has been designed such that the current can be given to the heater only in one sense. This prevents any positive feedback effect that can take place when a too small value of bias current is given to the thermistor, or the thermistor operating point is in the linear region of Fig. 6. Diode $D_{N}$ and resistor $\mathrm{R}_{\mathrm{N}}$ are added to limit a possible large negative excursion of the input of $\mathrm{A}_{\mathrm{G} 1}$.

The inclusion of the comparator "Com" was found to be very useful. Sometimes it happens that when the loop is closed the first time, a transient may be set and a large value of current is given to $\mathrm{R}_{\mathrm{h}}$. This can heat the system too much, with a consequent long recovery time. "Com" short circuits $R_{h}$ if the input to $\mathrm{A}_{\mathrm{G} 1}$ reaches large values, limiting the operating range to a safe region of operation.

The circuit that generates $g_{m}$ is the only part of the feedback added to the configuration of a standard front-end circuit used in CUORICINO. It has to be remarked that the operating point of thermistor $\mathrm{R}_{\mathrm{T}}$ can be chosen by simply exploiting the offset correcting circuit that equips any other channel. The correction is remotely made and a binary code is stored. This way if, for any reason, the system must be restarted, the offset binary code can be reused, allowing the same final stabilized temperature for the frame and the detectors.

Equation (15) accounts for the static behavior of the system. An estimation of the dynamic performance can be evaluated. First we have to calculate the quantity A of (7) that is:

$$
\begin{aligned}
\mathrm{A} & =\frac{\Delta \mathrm{P}_{\mathrm{EL}}}{\Delta \mathrm{R}}=2\left[\mathrm{~V}_{\mathrm{T}}-\mathrm{V}_{\mathrm{OFF}}\right] \mathrm{A}_{\mathrm{PGA}}^{2} \mathrm{~g}_{\mathrm{m}}^{2} \mathrm{R}_{\mathrm{h}} \frac{\mathrm{R}_{\mathrm{L}}}{\mathrm{R}_{\mathrm{T}}+\mathrm{R}_{\mathrm{L}}} \mathrm{I}_{\mathrm{T}} \\
& =\frac{\mathrm{R}_{\mathrm{L}}}{\mathrm{R}_{\mathrm{T}}+\mathrm{R}_{\mathrm{L}}} \frac{\mathrm{R}_{\mathrm{T}}-\mathrm{Z}_{\mathrm{T}}}{\mathrm{Z}_{\mathrm{T}}} \frac{\mathrm{K}_{\mathrm{F}}(\mathrm{T})}{\alpha}
\end{aligned}
$$

where use has been made of (15).

Now suppose that an input disturbing power, $\Delta \mathrm{P}_{\mathrm{n}}$, tries to heat the frame. From (8) we have:

$$
\begin{aligned}
& \Delta \mathrm{T}_{\mathrm{n}}=\frac{1}{j \omega \mathrm{C}_{\mathrm{F}}+\mathrm{K}_{\mathrm{F}} \frac{\mathrm{Z}_{\mathrm{T}}+\mathrm{R}_{\mathrm{L}}}{\mathrm{R}_{\mathrm{L}}+\mathrm{R}_{\mathrm{T}}} \frac{\mathrm{R}_{\mathrm{T}}}{\mathrm{Z}_{\mathrm{T}}}} \Delta \mathrm{P}_{\mathrm{n}} \\
& \stackrel{\mathrm{R}_{\mathrm{L}}}{\stackrel{\mathrm{R}_{\mathrm{T}}}{\mathrm{R}}} \frac{\mathrm{Z}_{\mathrm{T}}}{\mathrm{R}_{\mathrm{T}}} \frac{1}{\mathrm{j} \omega \frac{\mathrm{C}_{\mathrm{F}}}{\mathrm{K}_{\mathrm{F}}} \frac{\mathrm{Z}_{\mathrm{T}}}{\mathrm{R}_{\mathrm{T}}}+1} \frac{\Delta \mathrm{P}_{\mathrm{n}}}{\mathrm{K}} .
\end{aligned}
$$

The final expression is very simple. The feedback has the effect to reduce the thermal time constant and attenuate the temperature change consequent to a noisy input power by a factor of $Z_{T} / R_{T}$. In the case of CUORICINO this factor was calculated in the previous section to be about 0.009 . The heat capacity of the oxygen free copper frame of CUORICINO is $\mathrm{C}_{\mathrm{F}}(\mathrm{T})=$ $1.2 \times 10^{-5} \times 12000 \mathrm{~T}$, or about $1.4 \mathrm{~mJ} / \mathrm{K}$. The open loop time constant is $\mathrm{C}_{\mathrm{F}} / \mathrm{K}_{\mathrm{F}} \approx 100 \mathrm{sec}$. The feedback time constant is reduced to less than about $1 \mathrm{sec}$. The same applies also for the amount of temperature change.

There is a latter important aspect concerning the adopted system. From (15) it is possible to verify that the stabilized temperature may drift with $\mathrm{R}_{\mathrm{L}}$ and/or $\mathrm{V}_{\mathrm{OFF}}$ and/or $\mathrm{V}_{\mathrm{BIAS}}$. Nevertheless these 3 elements are of the same type of those used to operate any detector of the array. The consequence is that the stabilization system, on the average, compensates not only for the drift of the heat sink temperature, but also for the drift coming from these other possible contributing elements.

\section{EXPERIMENTAL RESULTS}

To reach the cryogenic temperature of about $7 \mathrm{mK}$ necessary to run CUORICINO, we use a dilution refrigerator. A gas mixture composed of ${ }^{3} \mathrm{He}-{ }^{4} \mathrm{He}$ is forced to circulate between room temperature and the cold finger, to which the detector frame is thermally connected with 2 copper strips. While circulating from room temperature down to the lower temperature the mixture is cooled by thermal contact with various thermal stages. The described process and the properties of ${ }^{3} \mathrm{He}-{ }^{4} \mathrm{He}$, a Fermion-Boson gas, allow reach the very low expected temperatures.

The first 2 stages at the higher temperatures, which are mainly responsible for the detector drift, are the main bath and the $1 \mathrm{~K}$ pot. The main bath is the Liquid He, $\mathrm{LHe}$, reservoir at $4.2 \mathrm{~K}$. The $1 \mathrm{~K}$ pot is a vessel that is filled with liquid from the main bath and is continuously pumped such that its temperature lowers to $1.2 \mathrm{~K}$. To maintain a constant level in the $1 \mathrm{~K}$ pot an equilibrium must be found between the filling and the pumping efficiency.

To compensate the LHe consumption we have used 2 methods. 1) The He gas evaporated from LHe in the main bath is driven to a liquefier to produce LHe that the liquefier itself injects back in the main bath; 2) a periodic refilling of the main bath is made by a human intervention. We used method 1) in the first part of our experiment, but after some mechanical problems we decided to use method 2), so removing the liquefier.

At the beginning of the CUORICINO run the stabilizing system was not in use. In Fig. 8 we can see the baseline drift of 5 detector channels, chosen to represent the typical observed behavior during a few days. Since the liquefier had not full efficiency, the level of the main bath lowered by about $2.2 \% /$ day. As a consequence the baseline of the detectors changes accordingly. Due to the good thermal sensitivity of our bolometers, this drift ranges between $50 \mathrm{keV} / \mathrm{day}$ to more than $150 \mathrm{keV} /$ day. In the period considered here the filling level of the $1 \mathrm{~K}$ Pot was very stable, about $-0.6 \% /$ day, see Fig. 9. The conversion in $\mathrm{keV}$ of the baseline drift corresponds to about $300 \mathrm{nK} / \mathrm{keV}$ and about $600 \mathrm{nK} / \mathrm{keV}$ of detector temperature change, depending on the crystal in the array $\left(5 \times 5 \times 5 \mathrm{~cm}^{3}\right.$ or 


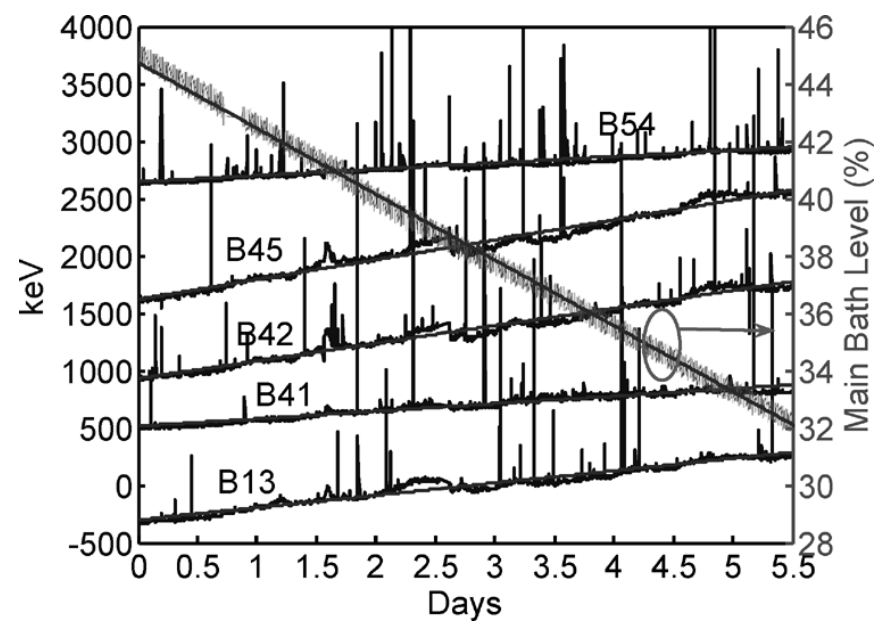

Fig. 8. Drift of 5 channels (labels indicating their convention name) of CUORICINO before system stabilization, left axis, the minimum drift is channel B54, $55 \mathrm{keV} /$ day; maximum drift is channel B45, $173 \mathrm{keV} /$ day. The main bath level is on the right axis, $-2.2 \%$ /day. An energy offset of $700 \mathrm{keV}$ has been added to the 4 upper channels to base legibility.

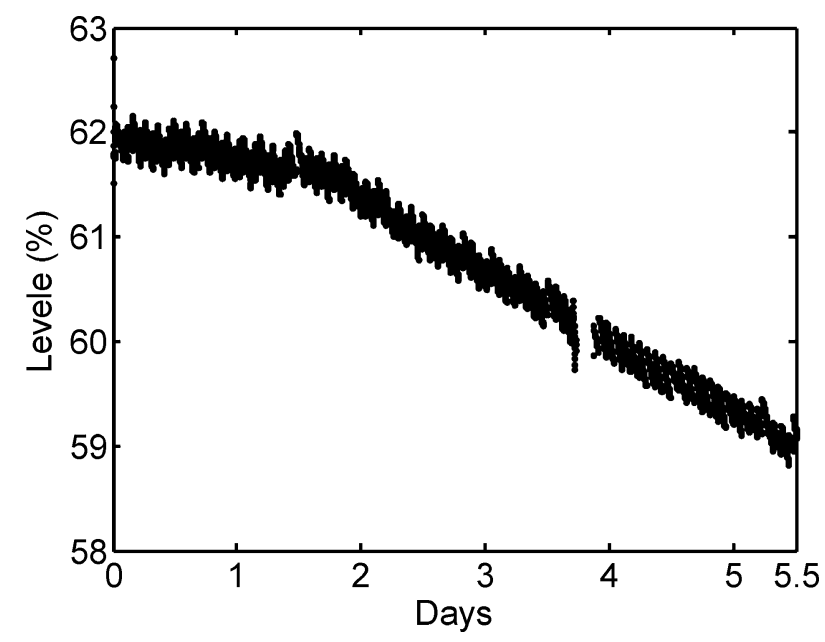

Fig. 9. $1 \mathrm{~K}$ pot level changes about $-0.6 \%$ /day during the period shown in Fig. 8 .

$3 \times 3 \times 6 \mathrm{~cm}^{3}$ ). From Fig. 8 we have that the temperature drift is close to $15 \mu \mathrm{K} /$ day to $45 \mu \mathrm{K} /$ day for the larger detectors.

Things became completely different as soon as the stabilization system was introduced. In Fig. 10 the drift of the same set of detectors is shown during a similar time period. The baseline drift is harder to detect. As an upper limit we quote it between $-0.02 \mathrm{keV} /$ day $(6 \mathrm{nK} /$ day), for the best situation, and -0.84 $\mathrm{keV} /$ day $(250 \mathrm{nK} /$ day) at worst. If we consider the fact that in the same period the main bath level dropped-out by about $-0.93 \% / d a y$, the reduction of the drift was never less than a factor of 70 and even more than a factor of 200 (2000 for the very best case) with respect to those of the prestabilization period.

It has to be remarked that while stabilization was used, the main bath level had small variations and the $1 \mathrm{k}$ Pot emptied daily, Fig. 11. This is the reason why the main bath level of Fig. 10 is not a straight line. It underwent to small periodic changes caused by the complete filling of the $1 \mathrm{k}$ Pot. The stabilization system was also able to reject this effect.

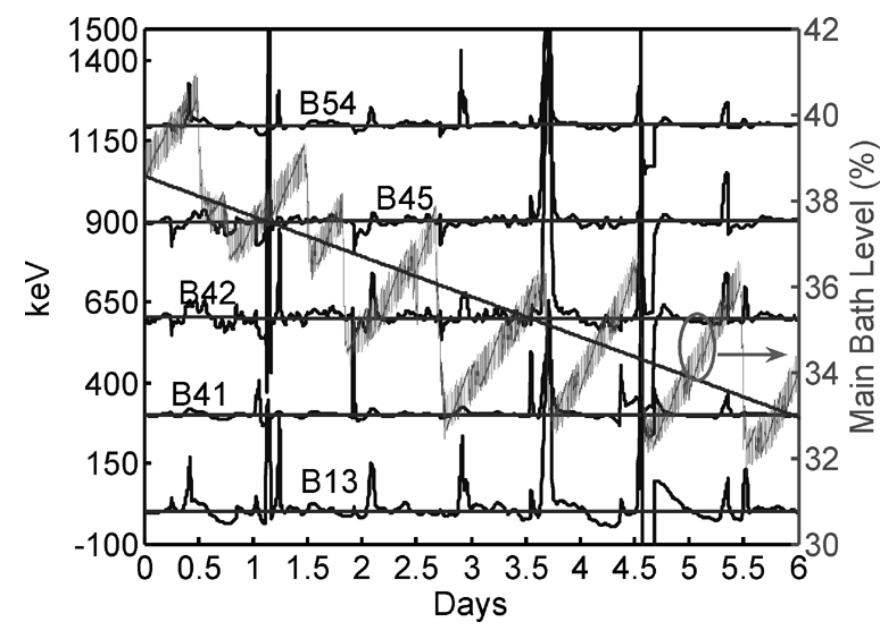

Fig. 10. Baseline of the selected channels, the labels indicate their convention name, after stabilization. An energy offset of $300 \mathrm{keV}$ has been added to the 4 upper channels to guide the eyes. The right axis is the liquid level inside the main bath. The smaller drift is from channel B13, $-0.02 \mathrm{keV} / \mathrm{day}$, the larger from B42, $-0.84 \mathrm{keV/day}$. The main bath level drop was about $-0.94 \% /$ day.

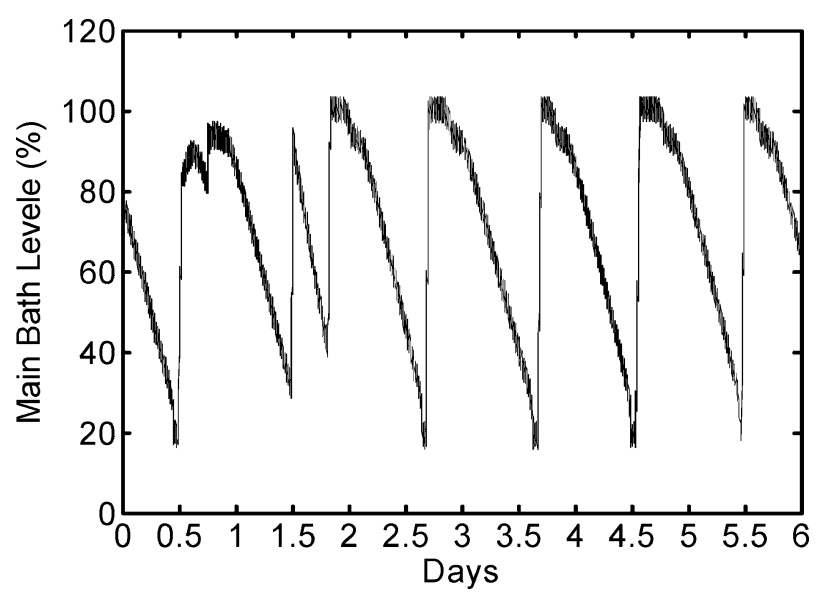

Fig. 11. $1 \mathrm{k}$ Pot level. A refill was necessary about any 1.5 days in the same period as that of Fig. 10.

A probe of the efficiency of the stabilization system is the present situation in which there is a complete refill of the main bath every two days. As it can be seen in Fig. 12, where another set of detectors is shown for generality, the average drift does not change during two successive fillings of the main bath, with respect to the previous filling method, also in this very extreme case. Fig. 13 shows a longer measurements period, in which the main bath is completely filled many times. Fig. 14 shows the level of the $1 \mathrm{~K}$ Pot during a similar time period of that of Fig. 13. Although it was refilled asynchronously with respect to the main bath, no effects are visible in the baseline shift. This result shows that there is a very high rejection of the drift of the refrigerating system by the use of the implemented instrument.

The large insensitivity of the detector baseline to the main bath level is the more remarkable result obtained. It allows the periodic complete refilling of the main bath which would otherwise not be possible without the stabilization system.

It has to be remarked that the quoted figure is not limited by the developed instrument, but from the sensitivity of the adopted 


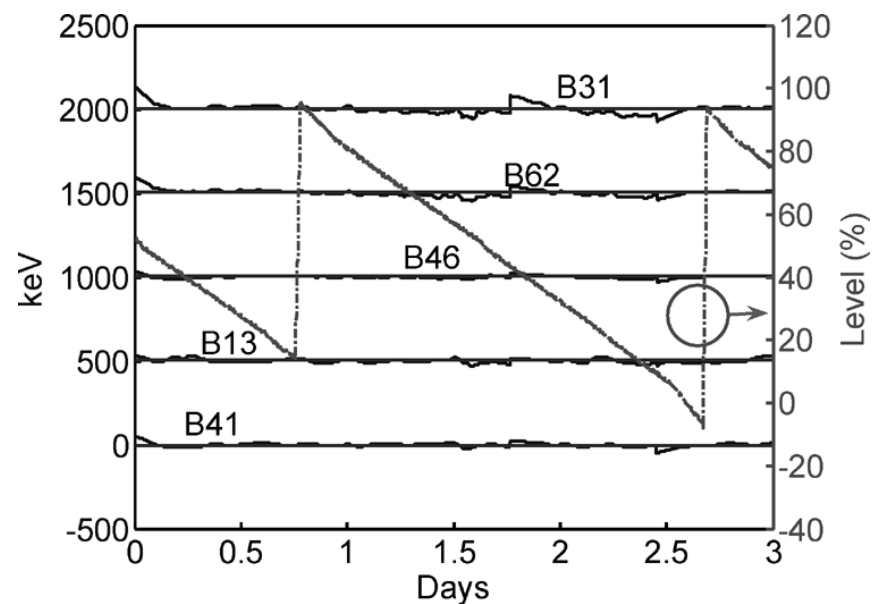

Fig. 12. The residual drift of a few detector channels, the labels indicate their convention name, without the presence of the liquefier and limited to two days, the period between the successive refill of the he main bath. The worst drift is 0.9 $\mathrm{keV} /$ day. An energy offset of $500 \mathrm{keV}$ has been added to the 4 upper channels to guide the eyes.

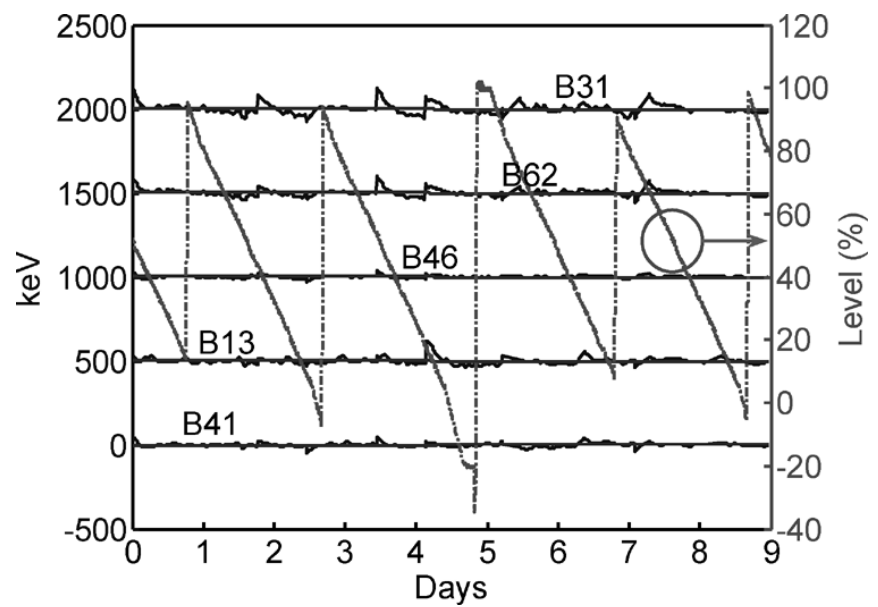

Fig. 13. The residual drift of a few detector channels, the labels indicate their convention name, without the presence of the liquefier and with refilling of the he main bath any 2 days. The observed drift is similar to that shown in Fig. 12. An energy offset of $500 \mathrm{keV}$ has been added to the 4 upper channels to guide the eyes.

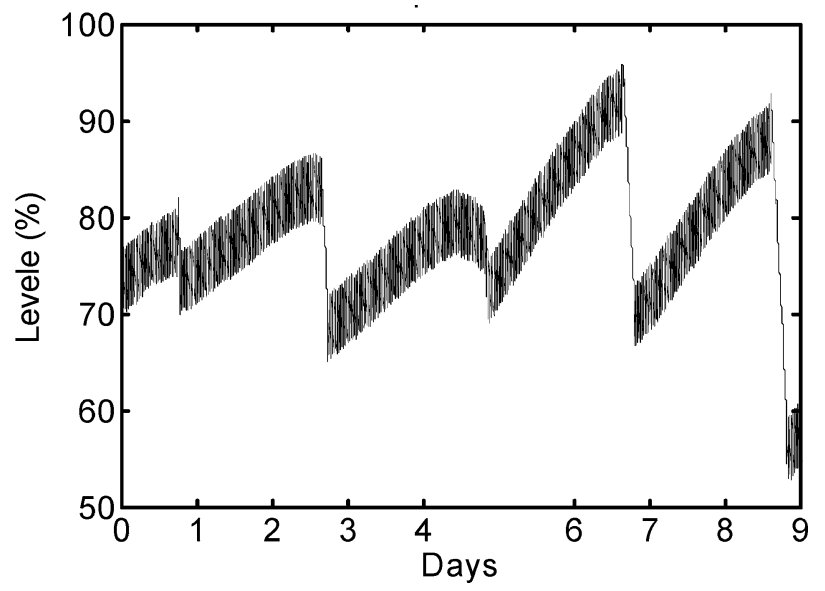

Fig. 14. The $1 \mathrm{k}$ Pot level during the same period of time of Fig. 13.

sensor elements. The rejection to the heat sink temperature drift of each bolometric channel has been found proportional to the ratio of the thermal sensitivity of the channel itself above that of the sensor used for the feedback loop, reflecting the spread present in the array.

Since our instrument exploits the same kind of sensor as those of the detector array, it is able to handle cases in which more sensitivity is provided by the detecting method.

\section{CONCLUSION}

A very simple circuit solution has been developed to implement the temperature stabilization of an array of large mass bolometric detectors. The circuit exploits the front-end to perform the task. Adding a very simple transconductance amplifier the final drift was reduced at least a factor of 70 with respect to the open loop condition. Many channels had even better improvement, of a factor of 200 and more. The final figure of merit is a drift better than $0.8 \mathrm{keV} /$ day, or about $250 \mathrm{nK} /$ day, for a full excursion of the level of the refrigerating liquid in the main reservoir, this figure being limited by the sensitivity of the thermal sensor used in the array.

Since the stabilization system operation exploits the same kind of equipment of any other detector of the array, a further figure of merit is the rejection of the room temperature drift on the circuit devices that compose the front-end.

Thank to the use of the front-end, the sensing element can be chosen similar to any other bolometer of the array, obtaining the same level of sensitivity in the temperature stabilization.

The developed instrument was introduced in the CUORICINO experiment in January 2004 and has been in use since then.

The modeling of the stabilization system has been done by reviewing the theory of operation of a bolometric system using the concept of a feedback network.

\section{ACKNOWLEDGMENT}

The authors thank Antonio Delucia and Giulio Galotta for the accurate preparation of the circuit boards and the electronics setup.

\section{REFERENCES}

[1] C. Arnaboldi, F. T. Avignone, J. Beeman, M. Barucci, M. Balata, C. Brofferio, C. Bucci, S. Cebrian, R. J. Creswick, S. Capelli, L. Carbone, O. Cremonesi, A. deWard, E. Fiorini, H. A. Farach, G. Frossati, A. Giuliani, P. Gorla, E. E. Haller, I. G. Irastorza, R. J. McDonald, A. Morales, E. B. Norman, A. Nucciotti, M. Pedretti, C. Pobes, V. Palmieri, M. Pavan, G. Pessina, S. Pirro, E. Previtali, C. Rosenfeld, S. Scopel, A. R. Smith, M. Sisti, G. Ventura, and M. Vanzini, "Physics potential and prospects for the CUORICINO and CUORE experiments," Astroparticle Phys., vol. 20, pp. 91-110, 2003.

[2] E. E. Haller, "Isotopically engineered semiconductors," J. Appl. Phys., vol. 77, pp. 2857-2878, 1995.

[3] G. Pessina, "A room temperature differential voltage sensitive preamplifier for large mass bolometric detectors," Nucl. Instrum. Methods Phys. Res. A, vol. 444, pp. 132-135, 2000.

[4] A. Alessandrello, C. Brofferio, C. Bucci, O. Cremonesi, A. Giuliani, B. Margesin, A. Nucciotti, M. Pavan, G. Pessina, and E. Previtali e Zen, "Methods for response stabilization in bolometers for rare decays," Nucl. Instrum. Methods Phys. Res. A, vol. 412, pp. 454-464, 1998.

[5] C. Arnaboldi, G. Pessina, and E. Previtali, "A programmable calibrating pulse generator with multi-outputs and very high stability," IEEE Trans. Nucl. Sci., vol. 50, no. 4, pp. 979-986, Aug. 2003. 
[6] C. Bucci, C. Arnaboldi, D. R. Artusa, F. T. Avignone, M. Balata, I. Bandac, M. Barucci, C. Brofferio, J. Beeman, S. Capelli, L. Carbone, S. Cebrian, O. Cremonesi, R. J. Creswick, H. A. Farach, A. Fascilla, E. Fiorini, G. Frossati, A. Giuliani, P. Gorla, E. E. Haller, I. G. Irastorza, R. J. McDonald, A. Morales, E. B. Norman, A. Nucciotti, E. Olivieri, V. Palmieri, E. Pasca, M. Pavan, M. Pedretti, G. Pessina, S. Pirro, C. Pobes, E. Previtali, M. Pyle, L. Risegari, C. Rosenfeld, M. Sisti, A. R. Smith, L. Torres, G. Ventura, and A. deWaard, "First results from the CUORICINO experiment," Nucl. Instrum. Methods Phys. Res. A, vol. 520, pp. 132-134, 2004

[7] T. Wilbanks, M. Devlin, A. E. Lange, S. Sato, J. W. Beeman, and E. E. Haller, "Improved low frequency stability of bolometric detectors," IEEE Trans. Nucl. Sci., vol. 37, no. 2, pp. 566-572, Apr. 1990.

[8] E. N. Glezer, A. E. Lange, and T. M. Wilbanks, "Bolometric detectors: Optimization for differential radiometers," Appl. Opt., vol. 31, pp. 7214-7218, 1992.

[9] C. Arnaboldi, C. Bucci, J. W. Campbell, A. Nucciotti, M. Pavan, G. Pessina, S. Pirro, E. Previtali, C. Rosenfeld, and M. Sisti, "The programmable front-end system for CUORICINO, an array of large-mass bolometers," IEEE Trans. Nucl. Sci., vol. 49, no. 5, pp. 2440-2447, Oct. 2002.

[10] E. E. Haller, "Advanced far-infrared detectors," Infrared Phys. Technol., vol. 35, pp. 127-146, 1994

[11] J. Zhang, W. Cui, M. Juda, D. McCammon, R. L. Kelley, S. H. Moseley, C. K. Stahle, and A. E. Szymkowiak, "Hopping conduction in partially compensated doped silicon," Phys. Rev. B, Condens. Matter, vol. 48, pp. 2312-2319, 1993

[12] M. Pedretti, "The single module for CUORICINO and CUORE detectors: Tests, construction and modeling," Phil.D. dissertation, Department of Chemical, Physical and Mathematical Sciences, Università degli Studi dell'Insubria, Como, Italy, 2004.
[13] R. C. Jones, "The general theory of bolometer performance," Opt. Soc. Amer, vol. 43, pp. 1-14, Jan. 1953.

[14] J. C. Mather, "Bolometer noise: Non equilibrium theory," Appl. Opt., vol. 21, pp. 1125-1129, Mar. 1982.

[15] — - "Bolometers: Ultimate sensitivity, optimization, and amplifier coupling," Appl. Opt., vol. 23, pp. 584-588, 1984.

[16] M. Galeazzi and D. McCammon, "Microcalorimeter and bolometer model," J. Appl. Phys., vol. 93, pp. 4856-4869, 2003.

[17] A. Alessandrello et al., "Signal modeling for $\mathrm{TeO} 2$ bolometric detectors," J. Low Temp. Phys., vol. 93, pp. 207-212, November 1993.

[18] A. Alessandrello, C. Brofferio, D. V. Camin, O. Cremonesi, A. Giuliani, M. Pavan, G. Pessina, and E. Previtali, "An electrothermal model for large mass bolometric detectors," IEEE Trans. Nucl. Sci., vol. 40, no. 4, pp. 649-656, Aug. 1993.

[19] L. Risegari, M. Barucci, C. Bucci, V. Fafone, P. Gorla, A. Giuliani, E. Olivieri, E. Pasca, S. Pirro, L. Quinteri, A. Rocchi, and G. Ventura, "Use of good copper for the optimization of the cooling down procedure of large masses," Cryogenics, vol. 44, pp. 167-170, 2004.

[20] C. Arnaboldi, C. Bucci, S. Capelli, A. Fascilla, P. Gorla, A. Nucciotti, M. Pedretti, G. Pessina, S. Pirro, E. Previtali, and M. Sisti, "The front-end readout for CUORICINO, an array of macro-bolometers and MIBETA, an array of $\mu$-bolometers," Nucl. Instrum. Methods Phys. Res. A, vol. 520, pp. 578-580, 2004.

[21] A. Alessandrello, C. Brofferio, C. Bucci, D. V. Camin, O. Cremonesi, A. Giuliani, A. Nucciotti, M. Pavan, G. Pessina, and E. Previtali, "A low do drift read-out system for a large mass bolometric detector," IEEE Trans. Nucl. Sci., vol. 44, no. 3, pp. 416-423, Jun. 1997. 